\title{
Highly effective peginterferon a-2a plus ribavirin combination therapy for chronic hepatitis $C$ in hemophilia in Korea
}

\author{
Suh Yoon Yang ${ }^{1}$, Hyun Woong Lee ${ }^{1}$, Youn Jae Lee ${ }^{2}$, Sung Jae Park ${ }^{2}$, Ki Young Yoo ${ }^{3}$, and Hyung Joon Kim ${ }^{1}$ \\ 1 Department of Gastrohepatology, Chung-Ang University Hospital, Chung-Ang University College of Medicine, Seoul; '2Department of \\ Gastrohepatology, Inje University Busan Paik Hospital, Inje University College of Medicine, Busan; ${ }^{3}$ Korea Hemophilia Foundation \\ Hospital, Seoul, Korea
}

Background/Aims: Chronic hepatitis C (CHC) is a major comorbidity in patients with hemophilia. However, there are no published data on the efficacy of antiviral therapy in Korea. We assessed the safety and efficacy of combination therapy with peginterferon a-2a plus ribavirin for $\mathrm{CHC}$ in hemophilia.

Methods: Patients ( $n=115)$ were enrolled between March 2007 and December 2008. Seventy-seven patients were genotype 1 or 6 , and 38 patients were genotype 2 or 3 . We evaluated rapid virologic responses (RVRs), early virologic response (EVRs), end-of-treatment response (ETRs), sustained virologic response (SVRs), and relapses. Safety evaluations included adverse events and laboratory tests.

Results: Eleven patients were excluded from the study because they had been treated previously. Among the remaining 104 treatment-naïve patients, RVR was achieved in 64 (60.6\%), ETR was achieved in 95 (91.3\%), and SVR was achieved in $89(85.6 \%)$. Relapse occurred in eight patients (8.9\%). Common adverse events were hair loss (56.7\%) and headache (51.0\%). Common hematologic adverse events were neutropenia (22.1\%), anemia (27.9\%), and thrombocytopenia (3.8\%). However, there were no serious adverse events such as bleeding. RVR was the only predictor of SVR in multivariate analysis.

Conclusions: Peginterferon a-2a plus ribavirin combination treatment produced a favorable response rate in CHC patients with hemophilia without serious adverse events. (Clin Mol Hepatol 2015;21:125-130)

Keywords: Chronic hepatitis C; Hemophilia; Peginterferon; Ribavirin

\section{INTRODUCTION}

Hepatitis C virus (HCV) infection is a major cause of morbidity and mortality in patients with hemophilia. More than $90 \%$ of hemophiliacs who received non-virucidally treated factor concentrates prior to 1986 were infected with HCV. Because of their need for pooled factor concentrates, fresh frozen plasma or cryoprecipitate, which uses blood from multiple donors, hemophiliacs have the highest rate of infection of all those at high-risk of infection. ${ }^{1,2}$ Therefore, in previous studies the prevalence of HCV infection in hemophiliacs was $40-90 \%$. $^{3-6}$

According to recent data, the prevalence of positive of anti-HCV

\footnotetext{
Abbreviations:

ALT, alanine aminotransferase; CHC, chronic hepatitis C; ETR, end of treatment response; EVR, early virologic response; $\mathrm{HBsAg}$, hepatitis B surface antigen; HBV, hepatitis B virus; HCV, hepatitis C virus; Hgb, hemoglobin; HIV, human immunodeficiency virus; RVR, rapid virologic response; SVR, sustained virologic response
}

\section{Corresponding author: Hyung Joon Kim}

Department of Gastrohepatology, Chung-Ang University Hospital, Chung-Ang University College of Medicine, 102 Heukseok-ro, Dongjakgu, Seoul 156-755, Korea

Tel: +82-2-6299-1399, Fax: +82-2-825-7571

E-mail:mdjoon@cau.ac.kr 
is $19.5 \%$ in hemophiliacs in Korea. ${ }^{7,8}$ However, there are no known reports of long-term treatment outcome including the late relapse. So we assessed the efficacy and safety of peginterferon $\alpha$-2a plus ribavirin therapy in hemophilic patients with chronic hepatitis $\mathrm{C}(\mathrm{CHC})$ in Korea.

\section{MATERIALS AND METHODS}

\section{Patients}

We enrolled one hundred and fifteen patients with hemophilia who were followed by the Korean hemophilia foundation hospital. The inclusion criteria were: 1) positive serum HCV RNA (>15 IU/mL) regardless of alanine transaminase (ALT) level during the six months before the study; and 2) aged eighteen years or older. Our exclusion criteria included the presence or history of any of the following: 1) decompensated liver disease, hepatocellular carcinoma or other malignant neoplastic disease; 2) liver transplantation; 3) severe cardiac or chronic pulmonary disease; 4) immunologically mediated disease; 5) retinopathy; or 6) poorly controlled psychiatric disease.

Liver biopsy was not required because the histological severity of hepatic disease was not a criteria for eligibility, and because this procedure is life threatening and very costly in hemophiliacs. Clinical evidence of cirrhosis was defined as signs of irregular margins of the liver on abdominal ultrasound or computed tomography (CT), endoscopic evidence of esophageal varices or hypertensive gastropathy, and/or enlarged portal vein or splenomegaly.

Some patients were naïve while others had previous treatment experience. However, we analyzed data of the treatment naïve patients because previous treatment experience could influence treatment outcomes.

A single unit of transfused factors was produced by extracting necessary factors from the blood of thousands of donors. Therefore, the duration of the infection was determined to start at the point of the first transfusion.

\section{Study design}

This study was designed as a single-center open-label one-arm observational study of peginterferon $\alpha-2$ a (Pegasys, Roche, Basel, Switzerland) and ribavirin (Copegus, Roche) combination therapy of $\mathrm{CHC}$ infection in hemophilia $\mathrm{A}$. The duration on enrollment was 22 months, from March 2007 to December 2008. Eligible patients received $180 \mu \mathrm{g}$ of peginterferon $\alpha$-2a subcutaneously once a week in combination with 800-1,200 mg of oral ribavirin per day, according to their genotype and body weight. Patients with virus genotypes 1 or 6 with a weight below $75 \mathrm{~kg}$ were given 1,000 mg of oral ribavirin per day, while patients over $75 \mathrm{~kg}$ received $1,200 \mathrm{mg}$ of oral ribavirin daily. Patients with virus genotypes 2 or 3 were given a constant ribavirin dose of $800 \mathrm{mg} /$ day. The treatment duration for genotype 1 or 6 infections was 48 weeks, and 24 weeks for patients with genotypes 2 or $3 .^{9}$

\section{Assessment of efficacy}

Hepatitis C virus genotyping was carried out using the restriction fragment mass polymorphism (RFMP) method. Viral load quantification was performed using the Cobas Amplicor HCV Monitor, v2.0 (Roche Diagnostics, Branchburg, NJ, USA) with a lower HCV RNA detection level of $15 \mathrm{IU} / \mathrm{mL}$.

Serum HCV RNA was measured at weeks 0, 4, 12, 24, 28 and 48 in genotypes 2 and 3 infections. In genotypes 1 and 6 , it was measured at weeks $0,4,12,48,52$ and 72 . Our primary end point was a sustained virologic response (SVR), defined as undetectable HCV RNA ( $<15 \mathrm{IU} / \mathrm{mL})$ at 24 weeks after cessation of treatment. Our secondary efficacy end points were rapid virological response (RVR: undetectable HCV RNA at week 4 of treatment), complete early virological response (CEVR: undetectable HCV RNA at week 12 of treatment), partial EVR (pEVR: detectable HCV RNA level but a decrease of $\geq 2 \log _{10}$ from the baseline level), end of treatment response (ETR: undetectable HCV RNA at the end of 24 or 48 weeks of treatment), null response ( $<2 \log _{10}$ reduction of HCV RNA from baseline at 12 weeks of therapy), and relapse (reappearance of HCV RNA in serum after therapy is discontinued). Patients with an insufficient virological response at 12 weeks (a detectable HCV RNA level and a decrease of $<2 \log _{10}$ from baseline) in genotype 1 or without ETR in all genotypes were considered to have treatment failure, and were withdrawn from the treatment. ${ }^{9}$

Three types of HCV relapse were considered: (1) early, defined as an HCV RNA rebound occurring between the end of treatment and week 12; (2) late, defined as an HCV RNA rebound occurring between weeks 12 and 24; and (3) very late, defined as an HCV RNA rebound occurring beyond week $24 .^{10}$

\section{Assessment of safety}

Safety was assessed by monthly laboratory tests and evaluation of adverse events during treatment and follow-up. Adverse events were graded by the investigators as mild, moderate, severe, or 
life-threatening, according to a modified World Health Organization grading system.

Non-life-threatening adverse events were managed by reduction of the dose of peginterferon or with a prescription of appropriate medications. The laboratory criteria for dose reduction of peginterferon were $500-750$ neutrophils $/ \mathrm{mm}^{3}$ or $30,000-50,000$ platelets $/ \mathrm{mm}^{3}$. Decline of neutrophil count below 500 cells $/ \mathrm{mm}^{3}$, decline of platelet count below $30,000 / \mathrm{mm}^{3}$ and decline of hemoglobin below $7 \mathrm{~g} / \mathrm{dL}$ (because of lack of tolerance) were considered as criteria for peginterferon discontinuation. The ribavirin dose was reduced when the hemoglobin level declined below $9 \mathrm{~g} /$ $\mathrm{dL}$ and was discontinued when it declined below $7.5 \mathrm{~g} / \mathrm{mL}$. When ever patients had abnormal laboratory test results, in addition to dose adjustment, they were asked to recheck that laboratory test at 1 or 2 weeks intervals until it improved. Otherwise, the treatment was discontinued permanently. ${ }^{9}$

\section{Statistical analysis}

Summary statistics for virological outcomes are reported for subgroups of patients defined by their baseline characteristics. Analyses include data from all patients who received at least one dose of study medication. Logistic regression was used in univariate and multivariate analyses to predict factors related to SVR. $P$ values of less than 0.05 were deemed to be significant. Continuous variables were analyzed using the Mann-Whitney $U$ test. In the tables, continuous variables are presented as median values (range, minimum value-maximum value), while qualitative and discrete variables are presented as absolute and relative frequencies as percentages. An intention-to-treat analysis was performed. All statistical analyses were conducted using SPSS version 14.0 for Windows (SPSS Inc., Chicago, IL, USA).

\section{Ethics statement}

The study protocol was approved by the institutional review board of Chung-Ang University College of Medicine (IRB No. 7-070-10-20). The data were analyzed anonymously.

\section{RESULTS}

\section{Patient characteristics at baseline}

Between March 2007 and December 2008, a total of one hun- dred and fifteen patients with hemophilia were enrolled and treated. From the all studied patients, one hundred and four patients were treatment-naïve and received HCV antiviral therapy for the first time. Eleven patients who were receiving retreatment, five patients were relapsed and six patients were null responder to previous standard interferon and ribavirin combination therapy. All patients were hemophilia A males. The median age of the patients was thirty-eight years at the beginning of therapy. The median body mass index (BMI) was 22.5 (range, 13.8-34.5) kg/m² and the median ALT level was 51.0 (16.0-308.0) IU/L. The median HCV RNA was 6.6 (3.7-8.2) $\log _{10} \mathrm{IU} / \mathrm{mL}$. Among probable HCV genotypes, genotype 1 was the most frequent (64\%) and the proportions of subtypes were $1 a(8 / 67), 1 b(54 / 67), 1 c(3 / 67)$ and $1 d$ (2/67). Thirty-five patients were genotype $2-2 a(23 / 35), 2 b(3 / 35)$, $2 \mathrm{a} 2 \mathrm{~b}$ (1/35), 2a2c (8/35)- and there was one patient of each genotype 3and 6 . In our study, the mean duration of infection was 25.1 years. Four patients had HBV-HCV co-infection and two patients had HIV-HCV co-infection. All HBV co-infections were negative for HBeAg and DNA undetectable and all HIV co-infections were in a stable state. Compensated cirrhosis was observed in one patient (Table 1).

\section{Virological response rates and treatment regimen efficacy}

Of the one hundred and four cases of HCV hemophilia, eightynine patients (85.6\%) achieved SVR. The one patient who had genotype 1 did not achieve cEVR. Additionally, eight patients $(7.7 \%)$ were prematurely withdrawn from treatment because of adverse events: five patients had a general weakness, one patient

Table 1. Baseline characteristics

\begin{tabular}{lc}
\hline Variables & $\mathbf{n = 1 0 4}$ \\
\hline Male $(\%)$ & $104(100)$ \\
Age $(\mathrm{yr})$ & $38(23-73)$ \\
BMI $\left(\mathrm{kg} / \mathrm{m}^{2}\right)$ & $22.5(13.8-34.5)$ \\
\hline ALT $(\mathrm{IU} / \mathrm{L})$ & $51.0(16.0-308.0)$ \\
HCV RNA $\left(\log _{10} \mathrm{IU} / \mathrm{mL}\right)$ & $6.6(3.7-8.2)$ \\
HCV genotype 1:2/3:6 & $67: 36: 1$ \\
\hline Duration of infection $(\mathrm{yr})$ & $25.1(10-40)$ \\
\hline HBV coinfection $(\%)$ & $4(3.8)$ \\
HIV coinfection (\%) & $2(1.9)$ \\
Liver cirrhosis (\%) & $1(1.0)$ \\
\hline
\end{tabular}

$\mathrm{BMI}$, body mass index; ALT, alanine transaminase; HCV, hepatitis C virus. 
had jaundice (bilirubin increased to $18 \mathrm{mg} / \mathrm{dL}$ ), one patient had an infection and one suffered from dizziness. Relapse occurred in eight patients (8.9\%). Five were early relapsers and one was a late relapser (Fig. 1). Two patients relapsed during the very late period.

Figure 2 shows the virological response by treatment duration for each genotype. As shown, the SVR rate of all patients was $85.6 \%(82.4 \%$ in genotypes $1 / 6$ and $91.7 \%$ in genotypes $2 / 3)$. The EVR rate was similar between these two groups $(P>0.05)$. However, we found that the RVR rate was significantly lower in genotype 1 and genotype 6 .

In univariate analysis, the baseline level of HCV RNA and

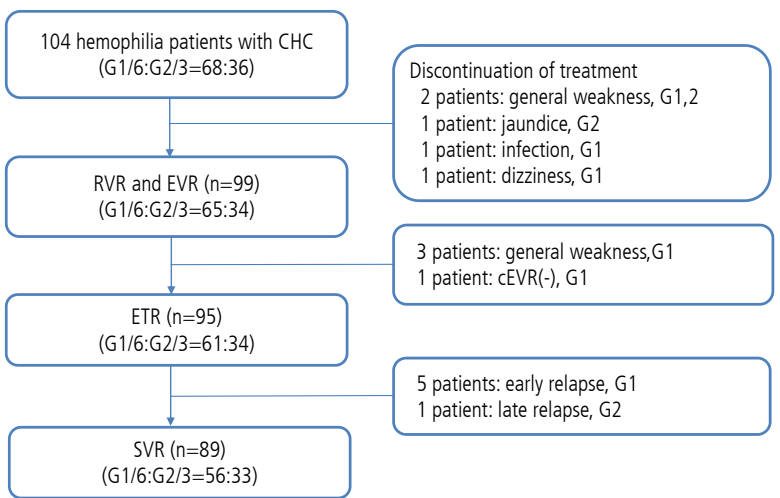

Figure 1. Clinical outcomes. CHC, chronic hepatitis $C_{;}$RVR, rapid virologic response; EVR, early virologic response; ETR, end-of-treatment response; SVR, sustained virologic response; CEVR, complete early virological response. achievement for RVR were predictive factors for SVR. However, in multivariate analysis, only achievement for RVR was identified as an independent predictor of SVR (Table 2). In addition, virological response in previous treatment-failure patients is shown in Table 3.

\section{Drug doses and side effects}

Adverse events are reported for the entire study population. The most common adverse events were hair loss, headache, itching, neutropenia and fatigue (Table 4). Most were mild, and only a few patients had severe hematologic events. The proportion of patients with severe neutropenia (a neutrophil count of $<500 / \mathrm{mm}^{3}$ )

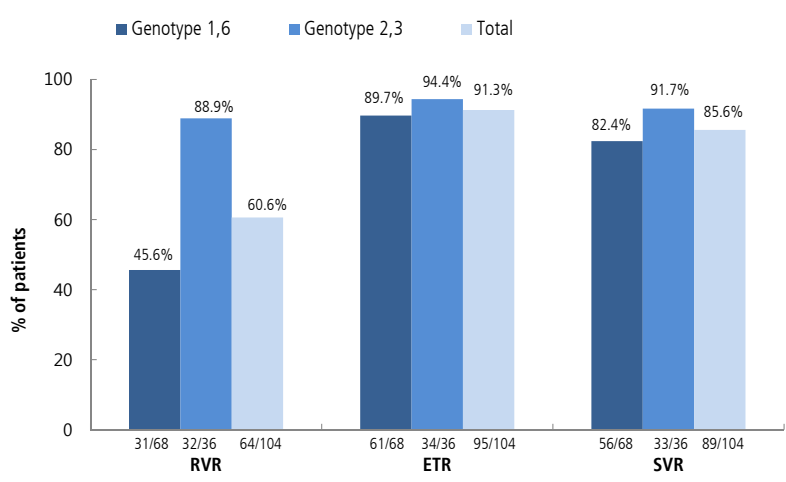

Figure 2. Virological response in treatment-naïve patients. Note: Response was determined based on the results of a treatment period using an intention-to-treat analysis. RVR, rapid virologic response; ETR, end-oftreatment response; SVR, sustained virologic response.

Table 2. Predictive factors for SVR

\begin{tabular}{|c|c|c|c|c|c|c|}
\hline \multirow[b]{2}{*}{ Variables } & \multicolumn{3}{|c|}{ Univariate analysis } & \multicolumn{3}{|c|}{ Multi-variate analysis } \\
\hline & $\begin{array}{c}\text { SVR } \\
(n=89)\end{array}$ & $\begin{array}{c}\text { No-SVR } \\
(n=15)\end{array}$ & $P$-value & Odds ratio & $95 \% \mathrm{Cl}$ & $P$-value \\
\hline Baseline HCV RNA ( $\left.\log _{10} \mid \mathrm{U} / \mathrm{mL}\right)$ & $6.4(3.7-8.2)$ & $7.0(6.3-8.0)$ & 0.009 & 0.460 & $0.192-1.100$ & 0.081 \\
\hline RVR, yes:no (\%) & $60: 29(67.4: 32.6)$ & $3: 12(20: 80)$ & 0.001 & 5.719 & $1.433-22.825$ & 0.014 \\
\hline Genotype (1/6:2/3, \%) & $56: 33(62.9: 37.1)$ & $12: 3(80: 20)$ & 0.250 & & & \\
\hline Age (yr) & $37(23-73)$ & $44(28-73)$ & 0.095 & & & \\
\hline BMI $\left(\mathrm{kg} / \mathrm{m}^{2}\right)$ & $22.4(13.8-34.5)$ & $22.9(16.9-31.6)$ & 0.577 & & & \\
\hline Baseline ALT (IU/L) & $52.0(17.0-248.0)$ & $47.0(16.0-308.0)$ & 0.150 & & & \\
\hline
\end{tabular}

SVR, sustained virologic response; HCV, hepatitis C virus; RVR, rapid virologic response; BMI, body mass index; ALT, alanine transaminase.

Table 3. Virological response in previous treatment-failure patients

\begin{tabular}{lccc}
\hline Pre-treatment result & $\mathbf{n = 1 1}$ & SVR (\%) & Cause of non-SVR \\
\hline Relapser & 5 & $5(100)$ & 1 - relapse at 3 months \\
Null responder & 3 & $2(75)$ & 1 - drop out at 10 weeks \\
\hline Drop-out & 3 & $2(75)$ & \\
\hline
\end{tabular}

SVR, sustained virologic response. 
Table 4. Safety profiles

\begin{tabular}{lccc}
\hline Variables & $\mathbf{n = 1 0 4 ( \% )}$ & Variables & $\mathbf{n = 1 0 4 ( \% )}$ \\
\hline Hair loss & $59(56.7)$ & Neutropenia $\left(/ \mathrm{mm}^{3}\right)$ & $45(43.3)$ \\
Headache & $53(51.0)$ & $750 \leq$ ANC $<1000$ & $22(21.2)$ \\
\hline Itching & $42(40.4)$ & $500 \leq$ ANC $<750$ & $17(16.3)$ \\
Fatigue & $42(40.4)$ & ANC $<500$ & $6(5.8)$ \\
Muscle ache & $40(38.5)$ & Anemia $(\mathrm{g} / \mathrm{dL})$ & $29(27.9)$ \\
Malaise & $35(33.7)$ & $8.0 \leq$ Hgb $<10.0$ & $21(20.2)$ \\
Fever & $31(29.8)$ & Hgb $<8.0$ & $8(7.7)$ \\
Rash & $30(28.8)$ & Thrombocytopenia $\left(/ \mathrm{mm}^{3}\right)$ & $4(3.8)$ \\
Poor appetite & $19(18.3)$ & $30,000 \leq$ Platelet $<50,000$ & $3(2.8)$ \\
Bleeding & $0(0)$ & Platelet $<30,000$ & $1(1.0)$ \\
\hline
\end{tabular}

ANC, absolute neutrophil count.

Table 5. Rates of discontinuation and dose reduction

\begin{tabular}{lc}
\hline Variables & $\mathbf{n = 1 0 4}(\%)$ \\
\hline Discontinuation due to serious adverse events & $8(7.7)$ \\
\hline RBV & $1(0.9)$ \\
\hline Peg IFN & $7(6.7)$ \\
\hline Dose reduction of RBV & $14(13.4)$ \\
\hline Anemia & $4(13.4)$ \\
\hline Dose reduction of peg IFN & $11(10.5)$ \\
\hline Neutropenia & $7(6.7)$ \\
\hline Thrombocytopenia & $3(2.9)$ \\
\hline General weakness & $1(0.9)$ \\
\hline
\end{tabular}

$\mathrm{RBV}$, ribavirin; peg IFN, peginterferon.

was $5.8 \%$, anemia $(\mathrm{Hgb}<8 \mathrm{~g} / \mathrm{dL}) 7.7 \%$ and thrombocytopenia $\left(<30,000 / \mathrm{mm}^{3}\right) 1 \%$. Moreover, no patients experienced bleeding.

The proportion of patients who discontinued the treatment due to a serious adverse event was $7.7 \%$ (Table 5). The discontinuation of ribavirin occurred in one patient $(0.9 \%)$ because of anemia and of peginterferon in seven patients $(6.7 \%)$ because of general weakness, jaundice and infection. However, there were no patient who discontinued for cytopenia. The proportion of patients who underwent peginterferon-dose reduction was 10.5\%: 6.7\% for neutropenia, $2.9 \%$ for thrombocytopenia, and $0.9 \%$ for general weakness. The proportion of patients undergoing ribavirin-dose reduction was $13.4 \%$ (for anemia).

\section{Virological response rates of coinfected patients}

All of the HBV coinfected patients achieved SVR. All of them were hepatitis $B$ carriers with a DNA undetectable state before and after the treatment. Only one patient showed a slight increase in the amount of virus after treatment, but it was still less than 10,000 copies $/ \mathrm{mL}$.

Of the two HIV coinfected patients, one patient achieved SVR and the other was withdrawn from treatment because of jaundice.

\section{DISCUSSION}

To our knowledge, this is the first study of long term clinical outcomes in patients with hemophilia and CHC treated with peginterferon plus ribavirin in Korea.

The standard treatment for $\mathrm{CHC}$ in 2008 was weekly subcutaneous peginterferon- $\alpha$ and daily oral ribavirin., ${ }^{9,11}$ The SVR rate was observed in $42-74 \%$ in hemophiliacs treated with the standard regimen (33-51\% in genotype 1 patients, $71-86 \%$ in genotype 2 or 3 patients. ${ }^{1,3,12-14}$ Results for non-hemophiliacs were similar (42-52\% in genotype 1, 76-84\% in genotypes 2 or 3). ${ }^{13,15}$

In our study, the SVR rate was $85.2 \%$ (81.8\% in genotype 1 , $92.1 \%$ in genotypes 2 or 3 ), higher than previous data. There were several reasons for the high SVR. First, there was good compliance. Because hemophilia is a rare disease in Korea, patients who registered in the Korean hemophilic foundation hospital have a good compliance. Most patients were able to follow up to the end of treatment and to the present. Second, almost all patients were treated using a standard dose. There were more young people than most studies of non-hemophilic patients, and most of them could tolerate the side effects. Because they did not have severe complications such as fever or bleeding, most patients were treated for a sufficient period of time to the standard regi- 
men. Third, there were few patients with HIV co-infection. Genotype 1 and co-infection with HIV were strong predictors of a worse response to peginterferon therapy. ${ }^{1,12,16}$ Furthermore, the treatment of patients with HBV co-infection was effective.

After the end of treatment and during long term follow up for about four years (to the present), it was possible to evaluate the rate and timing of relapse. Relapse occurred in eight patients (8.9\%). Two relapsers occurred within 4 weeks of the end of treatment, three relapsers occurred within 12 weeks and one relapser occurred within 24 weeks. Two were very late relapsers, one at 40 weeks and the other at 72 weeks (both were genotype $1 \mathrm{~b}$ ). Few studies have mentioned relapsers treated with peginterferon plus ribavirin. In recent studies, the rate of relapse has been $0.9-22 \%{ }^{17.18}$

The $p E V R$ was not analyzed separately as a predictive factor of SVR because there was no statistical significance. pEVR occurred in four patients who had genotype $1 \mathrm{~b}$. Two of them did not achieve ETR, and the others relapsed.

A previous study showed that the favorable allele of IL-28 is more frequent in Asian patients compared to Caucasian patients, which may explain the superior response to interferon based therapies in Asian patients, including Koreans. ${ }^{5,18,19}$ To determine if this is indeed the case, we plan to perform single nucleotide polymorphism genotyping of IL-28 with our samples in the future.

There are limitations of this study. The primary limitation is the small number of patients. Considering the fact that Korea has few hemophiliacs, it was not be easy to enroll in the study. The other major limitation is that this is a retrospective cohort study.

In conclusion, we showed that responses to antiviral therapy in patients with hemophilia appear to be superior to those in the general population. There were no serious adverse events related to hemophilia, such as bleeding.

\section{Conflicts of Interest}

The authors have no conflicts to disclose.

\section{REFERENCES}

1. Patel $H$, Heathcote EJ. When to treat and the benefits of treating hepatitis C in patients with haemophilia. Haemophilia 2009;15:20-32.

2. Lee $C$, Dusheiko $G$. The natural history and antiviral treatment of hepatitis C in haemophilia. Haemophilia 2002;8:322-329.

3. Posthouwer D, Mauser-Bunschoten EP, Fischer K, Makris M. Treatment of chronic hepatitis $C$ in patients with haemophilia: a review of the literature. Haemophilia 2006;12:473-478.
4. Maor Y, Schapiro JM, Bashari D, Lurie Y, Safadi R, Segol O, et al. Treatment of hepatitis $C$ in patients with haemophilia - the Israeli National Hemophilia Center experience. Haemophilia 2008;14:336-342.

5. Sinn DH, Kim YJ, Lee ST, Gwak GY, Choi MS, Lee JH, et al. Association of a single nucleotide polymorphism near the interleukin-28B gene with response to hepatitis C therapy in Asian patients. J Gastroenterol Hepatol 2011;26:1374-1379.

6. Pereira GH, Mangini C. Treatment of chronic hepatitis C virus infection among Brazilian haemophiliacs. Braz J Infect Dis 2008;12:20-23.

7. KHP. 2009 Annual Report: Korean Hemophilia Foundation 2009:40.

8. KHP. 2013 Annual Report: Korean Hemophilia Foundation 2013

9. Liver) KTKAftsot. Guidelines for treatment for Chronic hepatitis C. In, 2013.

10. Medrano J, Barreiro P, Resino S, Tuma P, Rodríguez V, Vispo E, et al. Rate and timing of hepatitis $C$ virus relapse after a successful course of pegylated interferon plus ribavirin in HIV-infected and HIVuninfected patients. Clin Infect Dis 2009;49:1397-1401.

11. EASL. EASL Clinical Practice Guidelines: management of hepatitis C virus infection. J Hepatol 2011;55:245-264.

12. Franchini M, Mengoli C, Veneri D, Mazzi R, Lippi G, Cruciani M. Treatment of chronic hepatitis $C$ in haemophilic patients with interferon and ribavirin: a meta-analysis. J Antimicrob Chemother 2008;61:1191-1200.

13. Manns MP, McHutchison JG, Gordon SC, Rustgi VK, Shiffman M, Reindollar $\mathrm{R}$, et al. Peginterferon alfa-2b plus ribavirin compared with interferon alfa-2b plus ribavirin for initial treatment of chronic hepatitis C: a randomised trial. Lancet 2001;358:958-965.

14. Alavian SM, Tabatabaei SV, Keshvari M, Behnava B, Miri SM, Elizee PK, et al. Peginterferon alpha-2a and ribavirin treatment of patients with haemophilia and hepatitis C virus infection: a single-centre study of 367 cases. Liver Int 2010;30:1173-1180.

15. Fried MW, Shiffman ML, Reddy KR, Smith C, Marinos G, Gonçales $\mathrm{FL}$, et al. Peginterferon alfa-2a plus ribavirin for chronic hepatitis $C$ virus infection. N Engl J Med 2002;347:975-982.

16. Maor Y, Bashari D, Kenet G, Lalezari S, Lubetsky A, Luboshitz J, et al. Hepatitis $C$ at the israeli national hemophilia center. Haemophilia 2006;12:68-74.

17. Pearlman BL, Traub N. Sustained virologic response to antiviral therapy for chronic hepatitis C virus infection: a cure and so much more. Clin Infect Dis 2011;52:889-900.

18. Ge D, Fellay J, Thompson AJ, Simon JS, Shianna KV, Urban TJ, et al. Genetic variation in IL28B predicts hepatitis C treatment-induced viral clearance. Nature 2009;461:399-401.

19. Lyoo K, Song MJ, Hur W, Choi JE, Hong SW, Kim CW, et al. Polymorphism near the IL28B gene in Korean hepatitis C virus-infected patients treated with peg-interferon plus ribavirin. J Clin Virol 2011;52:363-366. 